\title{
Phase transitions in rotating neutron stars cores: back bending, stability, corequakes, and pulsar timing
}

\author{
J. L. Zdunik ${ }^{1}$, M. Bejger ${ }^{1}$, P. Haensel ${ }^{1}$, and E. Gourgoulhon ${ }^{2}$ \\ 1 N. Copernicus Astronomical Center, Polish Academy of Sciences, Bartycka 18, 00-716 Warszawa, Poland \\ e-mail: [jlz; bejger; haensel] acamk.edu.pl \\ 2 LUTH, UMR 8102 du CNRS, Observatoire de Paris, 92195 Meudon Cedex, France \\ e-mail: Eric.Gourgoulhon@obspm.fr
}

Received 27 September 2005 / Accepted 6 January 2006

ABSTRACT

\begin{abstract}
Aims. We analyze potentially observable phenomena during spin evolution of isolated pulsars, such as back bending and corequakes resulting from instabilities, which could result from phase transitions in neutron star cores.

Methods. We study these aspects of spin evolution of isolated compact stars by means of analytical models of equations of state, for both constant-pressure phase transitions and the transitions through the mixed-phase region. We use high-precision 2-D multi-domain spectral code LORENE for the calculation of the evolutionary sequences of rotating neutron stars. This allows us to search the parameter space for possible instability regions, and possible changes in the stability character of rotating stars with phase transitions in their cores.

Results. We determine the conditions on the density jump in constant-pressure phase transitions which leads to the back bending phenomena or to the existence of the unstable segments in the evolutionary sequences of spinning down isolated normal neutron stars. We formulate the conjectures concerning the existence of two disjoint families of non-rotating and rotating stationary configurations of neutron stars. To clarify the effect of rotation on the stability of neutron star we present the particular case of EOSs leading to marginal instability of static and rotating configurations: marginal instability point in non-rotating configurations continues to exist in all evolutionary spin-down tracks. We discuss the fate of rotating stars entering the region of instability calculating the change in radius, energy release, and spin-up associated with the corequake in rotating neutron star, triggered by the instability. The energy release is found to be very weakly dependent on the angular momentum of collapsing star.
\end{abstract}

Key words. dense matter - equation of state - pulsars: general - stars: neutron - stars: rotation

\section{Introduction}

The properties of the high-density matter in compact stars are now studied by means both of nuclear physics and astrophysics. Many different equations of state (EOS) were proposed in the literature, based on different theoretical models of dense matter. In particular, it has been postulated that at high enough density, matter contains hyperons, and that phase transitions to exotic phases of dense matter, such as meson (pion, kaon) condensates, or quark matter can occur in the dense compact star cores (for a recent review of possible states of matter in compact star cores see e.g. Glendenning 2000). Unfortunately, the lack of precise knowledge of strong interactions between hadrons in dense matter, as well as deficiencies and approximations plaguing the many-body calculations of the EOS prevent us from knowing the actual structure of neutron star cores. Terrestrial experiments cannot supply information on the properties of matter at density $\gtrsim 10^{15} \mathrm{~g} \mathrm{~cm}^{-3}$ expected at neutron star center. We can only hope that observations of neutron stars will provide us with constraints which will enable us to select a correct dense matter model, or at least to limit the number of acceptable dense matter theories.

Particularly interesting method of searching for the phase transition in neutron star cores via pulsar timing was proposed by Glendenning et al. (1997). As a pulsar spins down, its central density increases, and for a certain density a new phase of matter can appear. In the case considered by Glendenning et al., the new phase consisted of quark matter. The authors suggested, that the softening of the EOS, induced by the formation of the new dense phase, leads to a temporary spinup era, the phenomenon called back-bending. Originally, the name comes from nuclear physics, where the phenomenon of "back-bending" was observed in the systematics of the moment of inertia of excited states of rapidly rotating nuclei, see e.g., Ring \& Schuck (1980). The calculations of Glendenning et al. were performed within the slow-rotation approximation (Hartle 1967; Hartle \& Thorne 1968), supplemented with additional relations resulting from accounting for the rotational stretching 
and frame-dragging effects (Weber \& Glendenning 1991, 1992).

Several other authors (Heiselberg \& Hjorth-Hensen 1998; Chubarian et al. 2000) carried out their calculations of the backbending phenomenon using the slow rotation approximation of Hartle. However, as shown by Salgado et al. (1994), the Hartle method, when compared with results of exact 2-D numerical codes, breaks down for angular velocity close to the Keplerian one.

First calculations concerning back-bending based on exact 2-D code were performed by Cheng et al. (2002). These authors used the version of KEH code (Komatsu et al. 1989a,b), improved by Stergioulas \& Friedman (1995) (see also references therein). In their work, Cheng et al. focused on the role of the crust for the very existence of the back-bending. Indeed, as they show, even a slight change in the physical state of the crust (for example, a change in the crust-core transition pressure) may significantly affect the results. This shows that high precision is mandatory for reliable calculation of the back-bending phenomenon.

Another important article containing results based on 2-D computations with the Stergioulas \& Friedman (1995) code, was published by Spyrou \& Stergioulas (2002). They showed that the results obtained by Glendenning et al. (1997) are plagued by large numerical uncertainties. For example, the very same EOS as that used by Glendenning et al. (EOS from Table 9.2 of Glendenning 2000) did not yield the back-bending phenomenon at all in Spyrou \& Stergioulas (2002)! It became evident that the back-bending problem is much subtler than previously considered, and that it requires careful handling as well as high-precision 2-D computations. Also, Spyrou \& Stergioulas pointed out some errors in previous papers on backbending. For example, the formula for braking index must be corrected by taking into account the rotational flattening of the star (Sect. 6 in Spyrou \& Stergioulas 2002).

Most recently, Zdunik et al. (2004) showed that the backbending phenomenon can also occur for the EOSs different from the mixed-phase one. They also pointed out importance of the stability with respect to the axi-symmetric perturbations. The appearance of hyperons in the dense matter (Balberg \& Gal 1997) softens some EOSs so much, that pulsars losing angular momentum actually spin up during a period of time. Paradoxically, during this spin-up phase pulsars could lose a significant amount of their angular momentum.

It should be stressed that all previous works (except of Zdunik et al. 2004) considered back-bending as a feature of the the dependence of the moment of inertia, $I$, on the rotation frequency, $f \equiv 1 /$ rotation period: $I=I(f)$. As shown recently by Zdunik et al. (2004), this can easily lead to incorrect determination of the stability of rotating stars. Zdunik et al. (2004) pointed out that many cases claimed before to correspond to the back-bending, actually cannot be realized in nature because of the instability with respect to the axi-symmetric perturbations.

One of the aims of the present work is to determine reliably and precisely the stability regions on the back bending segments of the spin evolution tracks. In order to avoid any precision problem, and to investigate large and possibly complete parameter space, we will work with analytical EOSs of dense matter exhibiting a softening at supra-nuclear density. Two examples of softening by a phase transition will be considered. We will study EOSs with constant-pressure phase transitions, characterized by a density jump obtained using the Maxwell construction. Second, we will use EOSs with phase transition extending over a finite pressure range in which two pure phases coexist forming a mixed-phase state. Such EOSs are obtained for the first-order phase transition between two pure phases by relaxing the condition of local electric charge neutrality and replacing it by less stringent condition of the global neutrality (Glendenning 1992). Mixed-phase state can be realized provided the surface tension at the interface between the two pure phases is not too large.

The plan of the article is as follows. In Sect. 2 we present various types of analytical EOSs used in the calculation of the spin evolution tracks. Numerical methods used in exact 2-D calculations are briefly presented in Sect. 3. Our numerical results are described in Sect. 4. We first describe the general criteria for the back-bending and the stability for spinning-down stars. Then, the results for the EOSs with a mixed-phase segment are studied in Sect. 4.1, and those with constant pressure phase transition with density jump are reviewed in Sect. 4.2. In Sect. 5 we describe a link between the existence of unstable segments in the families of static and rotating configurations of neutron stars. Change in neutron star parameters, accompanying transitions between two rotating configurations, triggered by instabilities of isolated rotating neutron stars, are studied in Sect. 6. Modifications in the pulsar timing and pulsar age evaluations, due to phase transitions in spinning-down isolated neutron stars are studied in Sect. 7. A summary of our results and their discussion is presented in final Sect. 8. Formulae referring to analytical models of EOSs with phase transitions are collected in the Appendix.

\section{Analytical EOS}

Studies of stability of stationary rotating configurations require high accuracy of the 2-D calculations. This is particularly true for the determination of a critical configuration which separates the stable configurations from the unstable ones. On the other hand, in our search for criteria which link the properties of the phase transition in dense matter with its effect on the stability of stellar configurations with new-phase cores we wanted to explore possibly large space of the phase transition parameters. In view of this, we decided to use analytic forms of the EOSs. In this way, the EOSs were easy to handle, and we could easily change the phase-transition parameters in a continuous way, detecting with high precision the appearance of instability. Moreover, with our numerical methods of solving the hydrostationary equilibrium problem, we had no unnecessary preoccupation with control of precision (see Sect. 3).

\subsection{Constant pressure phase transition}

Normal phase is described by a polytrope of index $\Gamma_{\mathrm{A}}$. The phase transition to a dense phase takes place at a specific pressure, $\mathrm{P}_{\mathrm{AB}}$, at which normal phase of baryon density $n_{\mathrm{A}}$ coexists with dense phase of baryon density $n_{\mathrm{B}}$, with baryon density 


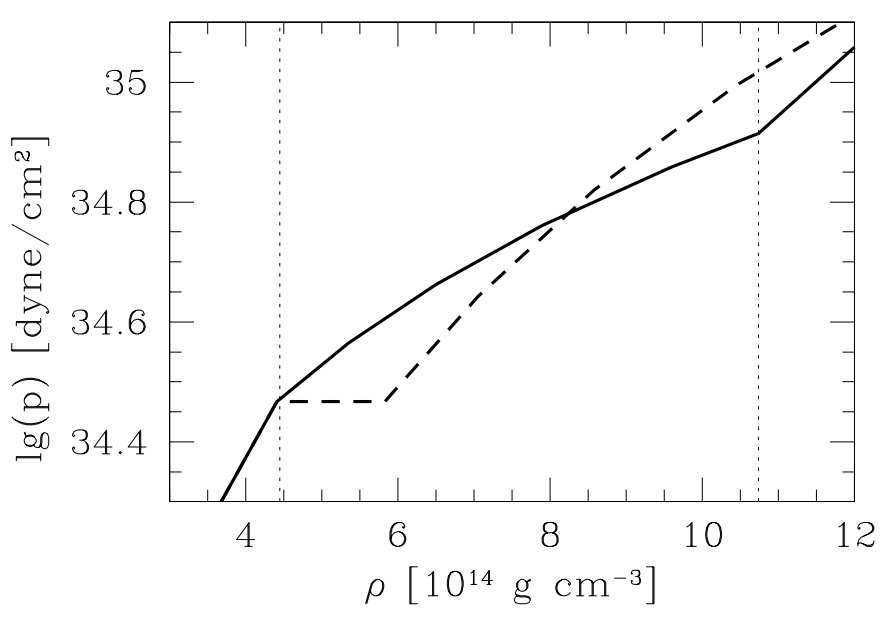

Table 1. Main parameters of the EOSs with mixed-phase segment. Below the mixed-phase transition point $n_{1}$ a polytropic EOS with $\Gamma_{\mathrm{A}}$ is used. Mixed phase extends within $n_{1}<n_{\mathrm{b}}<n_{2}$, and is described by a polytrope with adiabatic index $\Gamma_{\mathrm{m}}$. Above the density $n_{2}$ we assume pure quark matter with MIT bag model EOS $p=\frac{1}{3}\left(\rho-\rho_{0}\right) c^{2}$. In all cases the dimensionless polytropic pressure coefficient $K$ was equal 0.025 (see Appendix A for details). $M_{\mathrm{b}, \max }^{\mathrm{stat}}$ and $M_{\mathrm{max}}^{\text {stat }}$ denote the maximum allowable baryon and gravitational mass of the non-rotating star. The EOSs are labeled as follows: MSt produces a stable back bending, MUn - an unstable one, and MM produces a marginally stable case (for more details see the text).

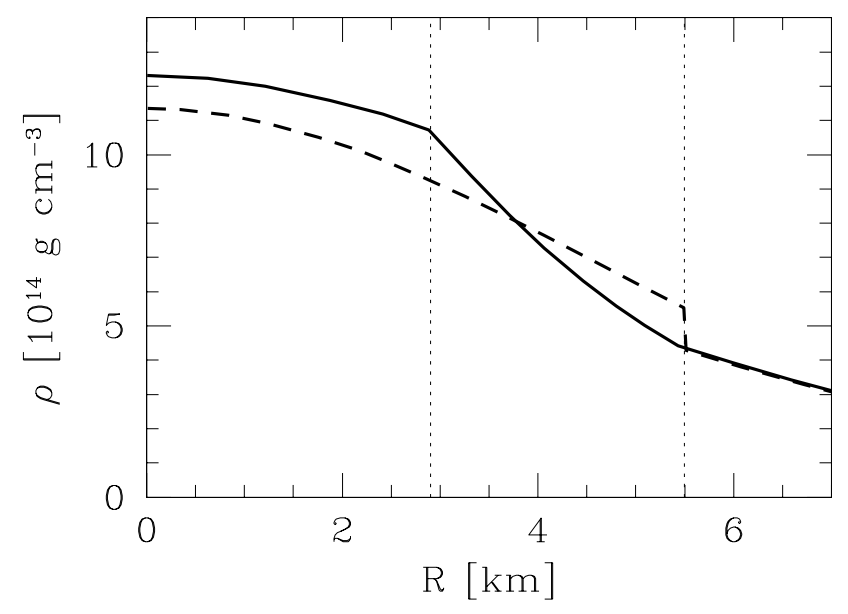

\begin{tabular}{cccccc}
\hline \hline EOS & $\Gamma_{\mathrm{A}}$ & $\begin{array}{c}n_{1} \\
{\left[\mathrm{fm}^{-3}\right]}\end{array}$ & $\Gamma_{\mathrm{m}}$ & $\begin{array}{c}n_{2} \\
{\left[\mathrm{fm}^{-3}\right]}\end{array}$ & $\begin{array}{c}M_{\mathrm{b}, \max }^{\text {stat }} \\
{\left[M_{\odot}\right]}\end{array}$ \\
\hline MSt & 2 & 0.35 & 1.5 & 0.8 & $1.508(1.393)$ \\
MUn & 2.5 & 0.2 & 1.3 & 0.65 & $1.586(1.453)$ \\
MM & 2.25 & 0.25 & 1.25 & 0.57 & $1.685(1.534)$ \\
\hline
\end{tabular}

Fig. 1. Two examples of EOSs employed in our calculations: constantpressure phase transition (dashed line, EOS with $\Gamma_{\mathrm{A}}=\Gamma_{\mathrm{B}}=2.25$, the density jump $\lambda=1.3$, can be found in Table 2) and transition through a mixed-phase state (solid line, thin dotted lines mark $n_{1}$ and $n_{2}$; EOS MM of Table 1). Top panel: $p(\rho)$ diagrams. Bottom panel: density profiles in stellar core, for a $M=1.33 M_{\odot}$ neutron star models.

jump $n_{\mathrm{B}}-n_{\mathrm{A}}>0$. Coexistence, equivalent to phase equilibrium, corresponds to equality of baryon chemical potential of both phases. The high-density phase B is a polytrope of in$\operatorname{dex} \Gamma_{\mathrm{B}}$. Details of the construction of the analytical EOSs are given in the Appendix A.1.

\subsection{Phase transition via the mixed-phase state}

Normal phase is described by a polytrope of index $\Gamma_{\mathrm{A}}$. Mixed phase starts at baryon density $n_{1}$, and ends at $n_{2}$; it has a polytropic EOS with index $\Gamma<\Gamma_{\mathrm{A}}$. This approximation is rather good for a sizable lower-density part of the mixed-phase segment (see Bejger et al. 2005). To be specific, pure high-density phase, which exists at $n>n_{2}$, was assumed to be quark matter, with a bag-model EOS. Details of construction of the analytical EOSs are given in the Appendix A.2. Depending on the values of parameters, we obtained three possible types of the EOS with mixed phase region. First, we obtained EOSs which give exclusively stable models between the minimum and maximum allowable mass; they are called MSt (Mixed

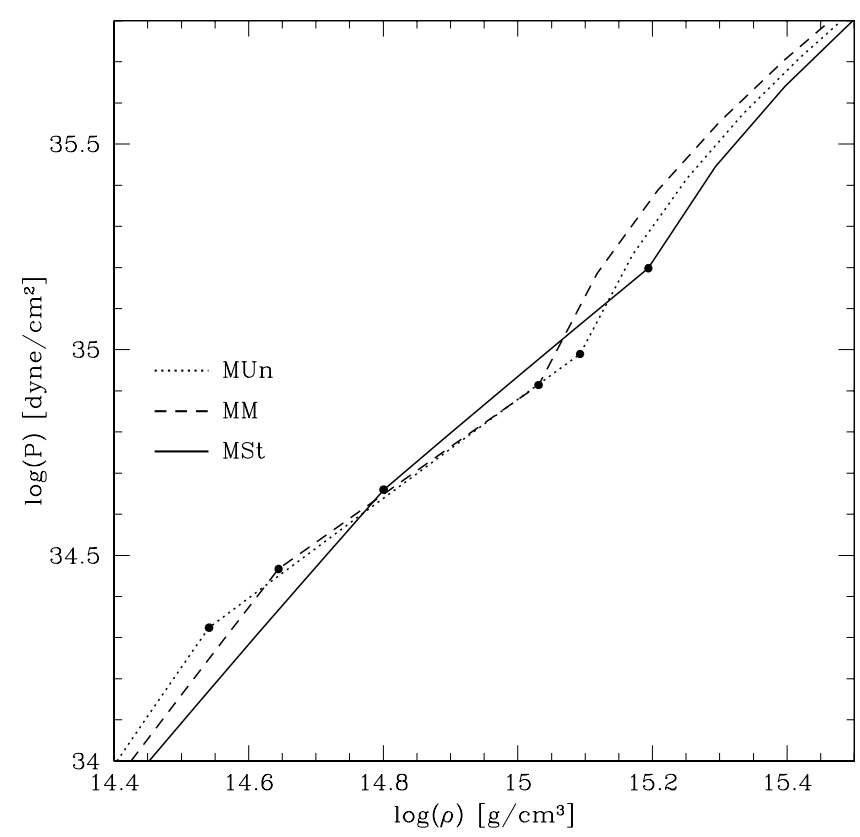

Fig. 2. Examples of the three EOSs with phase transition through the mixed-phase state, considered in the present paper. The parameters of the EOSs are given in Table 1.

Stable). Second, we obtained the EOSs which produced two distinct stable families of neutron stars, i.e., low density family and high density family. They are represented by two segments in the mass-central density plane and are separated by a segment of unstable configurations. Such an EOS is denoted by MUn (Mixed Unstable). There are also EOSs which yield a vanishingly short unstable segment of static configurations, so that the configurations are "marginally stable" there (an inflection point). This class of EOSs will be denoted by MM (Mixed Marginally Stable). Parameters of examples of analytical EOS of each of these three types are given in Table 1, and their $P(\rho)$ plots are shown in Fig. 2. These examples of the EOSs have $n_{1}$ similar to the models tabulated in Tables 9.2-9.3 by Glendenning (2000). Our values of $n_{2} \sim 3 n_{1}$ are systematically lower than those of Glendenning (2000) which are characterized by $n_{2} \sim 4 n_{1}$. However, this difference is not relevant 
for the general results of our analysis of stability of rotating stars.

\section{Numerical methods}

Hydrostationary stellar models have been computed by solving the Einstein equations for stationary axi-symmetric spacetime. The numerical calculations were performed by means of the rotstar code, a part of the LORENE public-domain objectoriented $\mathrm{C}++$ scientific library based on spectral methods. For the complete set of equations as well as the tests and the description of the numerical code we refer the reader to the articles by Bonazzola et al. (1993), Bonazzola et al. (1998) and Gourgoulhon et al. (1999). For the purpose of the present work, we have employed two (in the case of a constant-pressure phase transition) and three (phase transition via a mixed-phase state) spectral domains to describe the stellar interior and surface. The division into domains makes use of the adaptive coordinates while setting the boundaries between different phases. Therefore, the density field is smooth in each domain and the resulting accuracy is very high - in terms of GRV2 and GRV3 virial error indicators (see Nozawa et al. 1998) the relative error was on average $\sim 10^{-7}$.

\section{Back-bending and stability of rotating configurations}

As it has has been already mentioned by us in the previous paper treating specifically the back bending in hyperon stars (Zdunik et al. 2004), the back bending phenomenon itself is strictly connected with the existence of a minimum of $M_{\mathrm{b}}$ along the sequence of rotating configurations with fixed $f$. More generally, the softening of the EOS leads to the flattening of the $M\left(R_{\mathrm{eq}}\right)$ and $M\left(\rho_{\mathrm{c}}\right)$ curves representing these sequences. Here, $R_{\text {eq }}$ is the circumferential equatorial radius. The effect is larger for larger values of $f$, and it appears above some critical value of $f$. The softening of the EOS results then in a local maximum of $M_{\mathrm{b}}$. In such a case, there exists a region, close to the local maximum mass, in which the decrease of $J$ leads to the increase of $f$, and this is equivalent to back bending. The fragment of the curve, for which $M$ decreases as a function of $\rho_{\mathrm{c}}$, does not necessarily correspond to the instability region - the decrease at fixed $f$ does not imply the decrease at fixed $J$. Only the latter condition is equivalent to the instability of rotating configuration with respect to the axi-symmetric perturbations (Friedman et al. 1988). Summarizing, the particular shape of the $M\left(R_{\mathrm{eq}}\right)$ curve for a fixed $f$ indicates the presence of the back bending phenomenon, whereas the shape of $M\left(R_{\text {eq }}\right)$ at a fixed total angular momentum $J$ tells us if the configurations are stable.

In the present paper we will show examples of the EOS with constant-pressure (density-jump) phase transitions and mixed-phase transitions for which one of the two situations occurs:

- 1) all configurations are stable but the back bending exists;

- 2) the phase transition results in the instability region for rotating stars.
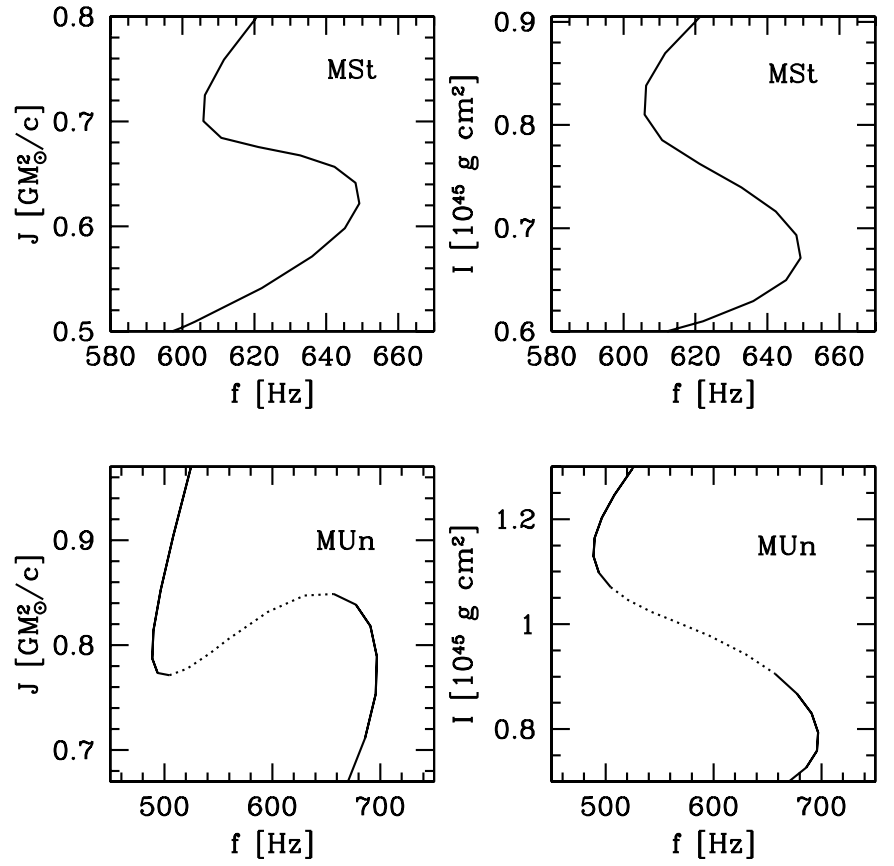

Fig. 3. Total angular momentum versus rotation frequency $f$ (left pan$e l s$ ), and moment of inertia $I \equiv J / \Omega$ versus $f$ (right panels), for EOSs MSt and MUn. The stability criterion is easily applied to left panels. It is clear that for the MSt EOS back bending feature is not associated with an instability, with all configurations being stable. On the contrary, the MUn EOS produces back bending with a large segment of unstable configurations. Simultaneously, the $I(f)$ curves for both EOSs are very similar and apparently show very similar back-bending shapes.

To distinguish between these two cases we can look at the behavior either of the curves $M_{\mathrm{b}}\left(\rho_{\mathrm{c}}\right)_{J}$ or $J(f)_{M_{\mathrm{b}}}$; the quantity fixed along a sequence is indicated by the lower index. For such curves the instability criterion directly applies. However, is not so easy to detect the instability using the $I(f)_{M_{\mathrm{b}}}$ plot, which may be similar to that corresponding to a fully stable sequence. An example is presented in Fig. 3, where we plot two functions: $J(f)_{M_{\mathrm{b}}}$ and $I(f)_{M_{\mathrm{b}}}$ for the two models of EOS (MSt and MUn) which are described in detail in Sect. 4.1. Upper panels correspond to the stable model MSt and lower to the MUn model for which a region of unstable configurations exists. The difference between the MSt and MUn cases is clearly visible in left panels $\left(J(f)_{M_{\mathrm{b}}}\right.$ curves), where we can easily recognize the instability region for MUn model, by applying the condition $\left(\mathrm{d} J / \mathrm{d} \rho_{\mathrm{c}}\right)_{M_{\mathrm{b}}}>0$, and keeping in mind that $\rho_{\mathrm{c}}$ is monotonic along this curve. However, the upper and lower right panels $\left(I(f)_{M_{\mathrm{b}}}\right)$ are quite similar, without any qualitative difference. Simultaneously, the back bending phenomenon in the MUn case is very large (almost by $200 \mathrm{~Hz}$ ), and obviously the phase transition results in an instability (increase of $J$ for increasing $\left.\rho_{\mathrm{c}}\right)$. And yet, $I\left(\rho_{\mathrm{c}}\right)$ is a monotonic decreasing function all the time. 

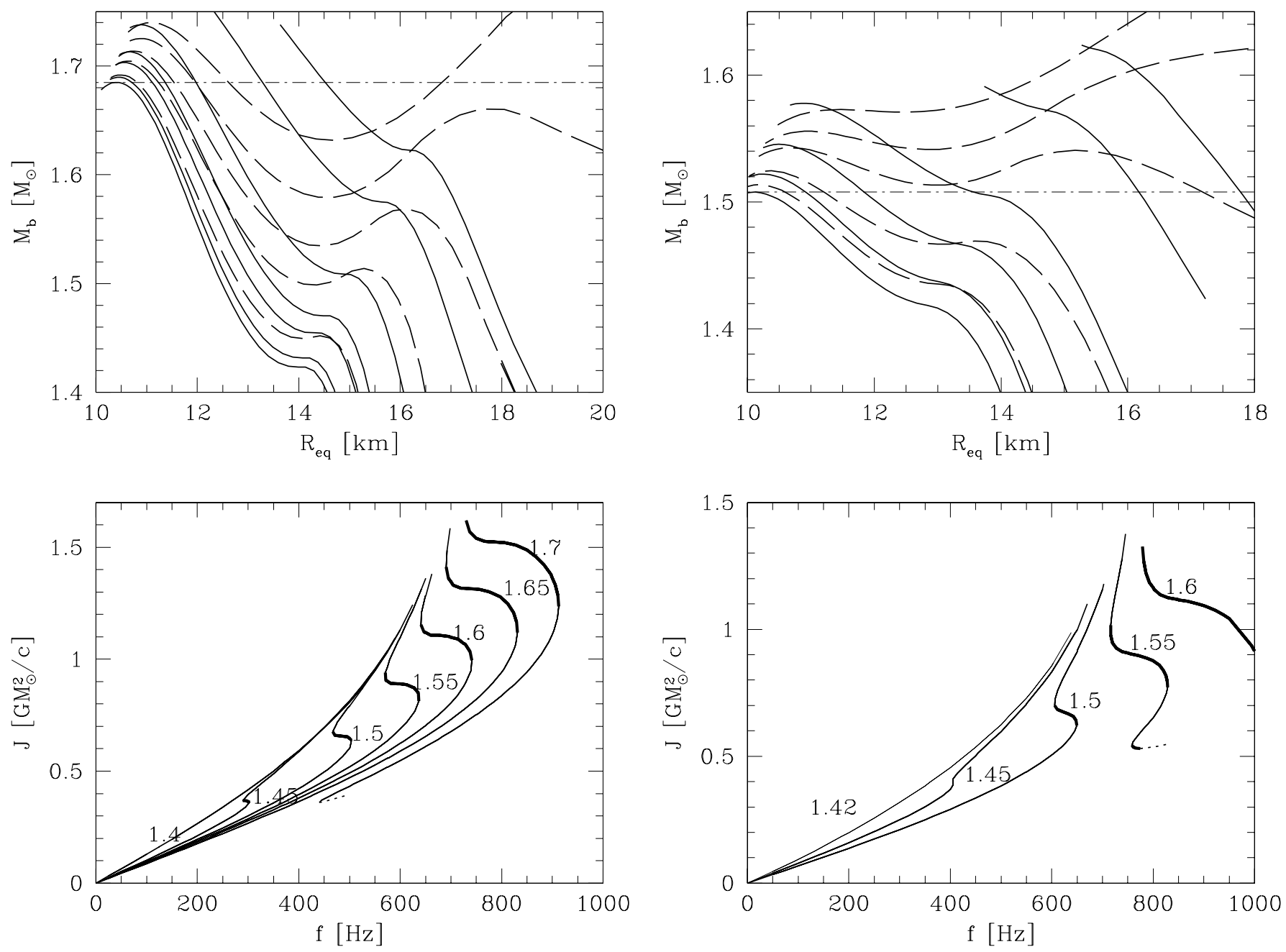

Fig. 4. Upper panel: stellar baryon mass vs radius for fixed frequency (dashed lines) and fixed total angular momentum (solid lines), for the model MM of mixed-phase EOS. Bottom panel: stellar angular momentum as a function of the rotational frequency for fixed baryon mass (indicated as a label, in solar masses) for the same MM EOS. This EOS corresponds to the marginal case from the point of view of stability - the curves $M\left(R_{\text {eq }}\right)_{J}$ and $J(f)_{M_{\mathrm{b}}}$ have flat horizontal regions. The regions of back-bending are drawn by thick lines.

\subsection{Transition to a mixed phase}

In Figs. 4-6 we present the results obtained for three different choices of the EOS's parameters describing mixed phase phase transitions (Table 1).

Model MM has been chosen to be a marginal one - there exists a region where the curve $M_{\mathrm{b}}\left(R_{\mathrm{eq}}\right)\left(\right.$ or $\left.M_{\mathrm{b}}\left(\rho_{\mathrm{c}}\right)\right)$ is locally horizontal, which means the marginal stability. The function $M_{\mathrm{b}}\left(R_{\mathrm{eq}}\right)$ for non-rotating $\operatorname{star}(f=0)$ has an inflection point slightly above the configuration where phase transition to the mixed phase occurs. At a fixed $f$, the condition for an inflection point reads,

$\left(\frac{\partial M_{\mathrm{b}}}{\partial \rho_{\mathrm{c}}}\right)_{f}=0, \quad\left(\frac{\partial^{2} M_{\mathrm{b}}}{\partial \rho_{\mathrm{c}}^{2}}\right)_{f}=0$.

In a special static case $(f=0)$ this condition is fulfilled for $M_{\mathrm{b}} \simeq 1.42 M_{\odot}$ and $R \simeq 14 \mathrm{~km}$. For this marginal EOS model,

Fig. 5. The same as in Fig. 4 but for the MSt EOS. Phase transition does not result in the stability loss - all configurations are stable.

the back bending phenomenon exists at all rotation rates, and for baryon masses larger than $1.42 M_{\odot}$. The region for which back-bending exists is bounded by the points corresponding to the local maxima and minima of the function $M_{\mathrm{b}}\left(R_{\mathrm{eq}}\right)$ at fixed rotational frequency $f$. In the marginal model for which the inflection-point condition, Eq. (1), is fulfilled for non-rotating stars, even an infinitesimally slow rotation leads to the minimum and maximum of the function $M_{\mathrm{b}}(R)_{f}$ (albeit infinitesimally close to each other), which is equivalent to the backbending (see dashed curves in Fig. 4). However, imposing rotation does not result in the instability - the functions $M_{\mathrm{b}}\left(\rho_{\mathrm{c}}\right)_{J}$ are monotonously increasing, independently of the rotation rate (solid curves in Fig. 4). Strictly speaking, for each $J$ there exists an inflection point of the function $M_{\mathrm{b}}\left(\rho_{\mathrm{c}}\right)_{J}$ (within the accuracy of our calculation). The size of back bending, defined as a frequency range for which $f$ increases for decreasing $J$ (and increasing $\rho_{\mathrm{c}}$ ), depends on the mass of the star (see bottom panel of Fig. 4). In the model MM, the back bending range varies from 0 (for $M_{\mathrm{b}} \leq 1.42 M_{\odot}$ ) to $\sim 180 \mathrm{~Hz}$ for the star with mass $M_{\mathrm{b}}=1.685 M_{\odot}$, equal to the maximum mass of non-rotating configurations (i.e. not supra-massive ones). 

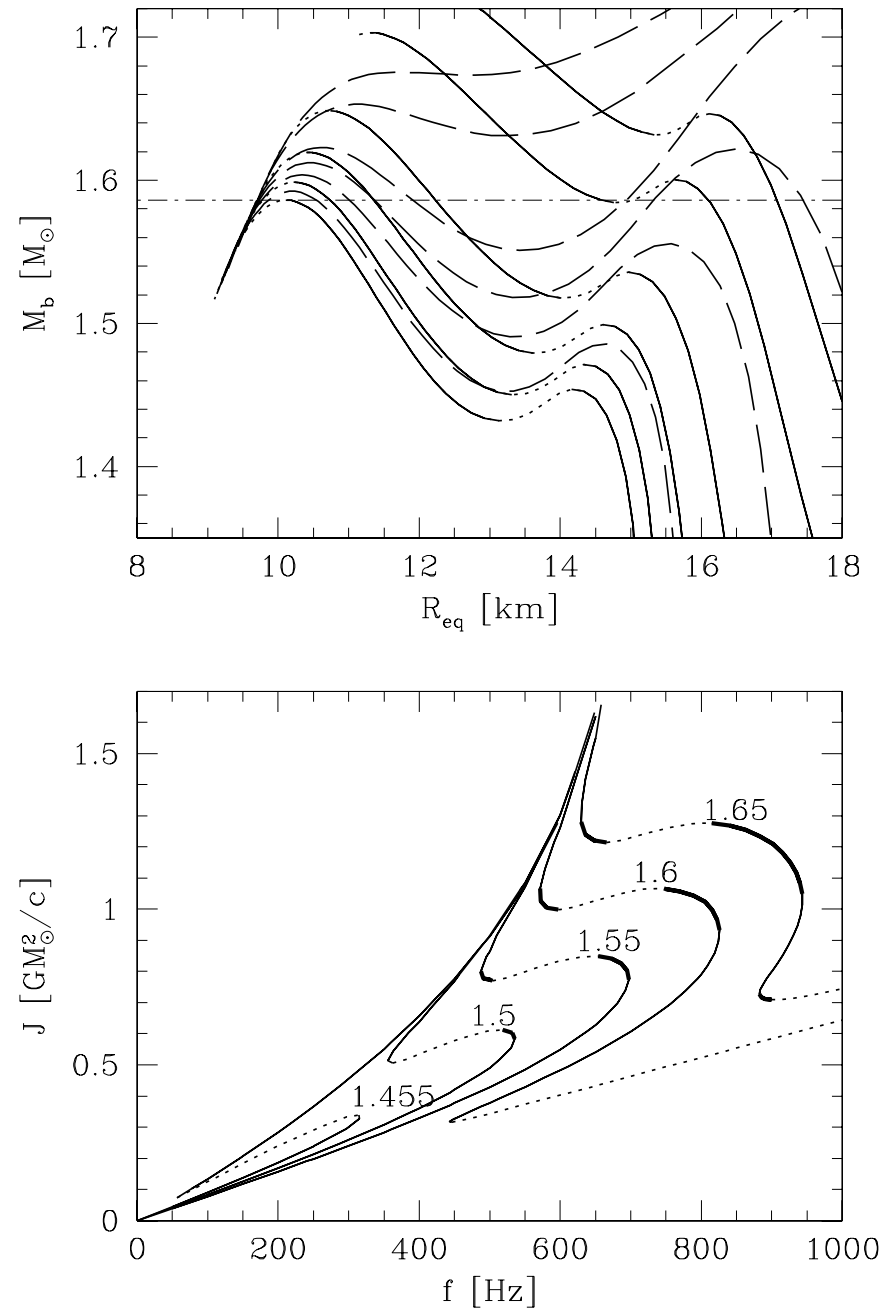

Fig. 6. The same as in Fig. 4 but for MUn EOS. Upper panel: Phase transition results in the stability loss, i.e., there exists a region where $M_{\mathrm{b}}$ decreases with increasing $\rho_{\mathrm{c}}$ at fixed $J$ (marked by dotted lines). Lower panel: plots in the $J-f$ plane, where on the dotted segments $J$ increases as $\rho_{\text {c }}$ increases (unstable configurations). Both features indicate instability with respect to the axi-symmetric perturbations.

Model MSt produces a set of stable configurations in the region of phase transition (Fig. 5). Here, $M_{\mathrm{b}}$ is an increasing function of $\rho_{\mathrm{c}}$ for non-rotating configurations, as well as along all rotating sequences of configurations at fixed $J$, terminating at the global maximum mass configurations. The back-bending phenomenon is in this case limited to the baryon masses larger than that for which the curve $M_{\mathrm{b}}\left(R_{\mathrm{eq}}\right)$ (or equivalently $M_{\mathrm{b}}\left(\rho_{\mathrm{c}}\right)$ ) at fixed $f$ has a flat, horizontal region (strictly speaking, where the Eq. (1) is fulfilled). The numerical values for the MSt model are $M_{\mathrm{b}}>1.45 M_{\odot}$ and $f>400 \mathrm{~Hz}$ (see for example the bottom panel of Fig. 5, where the curve for $M_{\mathrm{b}}=1.45 M_{\odot}$ has almost vertical part).

For the MUn model there exists a region for which configurations are unstable, i.e. the baryon mass is decreasing function of $\rho_{\mathrm{c}}$ at fixed $J$ (Fig. 6). In some sense this instability is not very strong - the difference between the maximum (local) and minimum mass is of the order of $0.3 \%$. However this feature (existence of instability region) is characteristic to all rotational frequencies - for all values of angular momentum $(J)$ fixed along the curve, the baryon mass has local maximum and local minimum connected by an unstable sequence of stellar configurations.

\subsection{Constant pressure phase transition}

For non-rotating configurations, the reaction of the star to a constant pressure (first order) phase transition has been studied in detail in the second half of 1980s (see Zdunik et al. 1987, and references therein). The appearance of a new, dense phase in the center of the star results in the change of the derivatives of the global stellar parameters with respect to $\rho_{\mathrm{c}}$ (see the formula B6 in the appendix of an article by Zdunik et al. 1987). Two important dimensionless parameters are: fractional density jump $\lambda=\rho_{\mathrm{B}} / \rho_{\mathrm{A}}$ and the relativistic parameter $x_{\mathrm{A}} \equiv P_{\mathrm{AB}} /\left(\rho_{\mathrm{A}} c^{2}\right)$. There exists a critical value of $\lambda, \lambda_{\text {crit }}=\frac{3}{2}\left(1+x_{\mathrm{A}}\right)$, such that for $\lambda>\lambda_{\text {crit }}$ configurations with an infinitesimally small B-phase core are unstable with respect to collapse into a new configuration with a large core of the dense phase. Putting it differently, a phase transition with $\lambda>\lambda_{\text {crit }}$ destabilizes the star at central pressure $P_{\mathrm{c}}=P_{\mathrm{AB}}$ at which the phase transition occurs. It should be stressed that while $\lambda<\lambda_{\text {crit }}$ guarantees stability of small-core configurations, it does not assure the stability of configurations with a finite, or - in an extreme case - a large core. In such a case the instability would result from the softness of the $\mathrm{B}$ phase somewhat above $\rho_{\mathrm{B}}$ and not directly from an over-critical $\lambda$. In other words the compressibility of a matter leads to the larger mean density in the core than the value $\rho_{\mathrm{B}}$ at the phase boundary. The response of the whole star to the appearance of the dense core built of the B-phase of the matter is determined by the mass and radius of this core (strictly speaking, this statement is true for non-rotating configurations, see Zdunik et al. (1987); for rotating ones also rotation rate and resulting oblateness play role). As a result even if $\lambda<\lambda_{\text {crit }}$ the first order phase transition can lead to the unstable configurations for finite size of the core. As a result for the given model of the matter in the phases A and B there exist the maximum value of density jump $\lambda_{\max }$ for which all configurations below maximum mass are stable. Of course $\lambda_{\max } \leq \lambda_{\text {crit }}$ and the difference between $\lambda_{\max }$ and $\lambda_{\text {crit }}$ is larger for softer EOS in the phase B.

Numerical results for a collection of sets of EOSs with constant pressure phase transition are collected in Table 2. The parameters presented in this table correspond to the onset of back bending, i.e., the rotational frequency and baryon mass for which the curve $M_{\mathrm{b}}\left(R_{\mathrm{eq}}\right)_{f}$ or $f(J)_{M_{\mathrm{b}}}$ starts to have a flat region. More precisely, at these values of frequency and mass, an inflection point appears in the curves under consideration. We also included parameters of those EOSs for which all nonrotating stars with $M_{\mathrm{b}}<M_{\mathrm{b}, \max }^{\text {stat }}$ are stable. This means that for such EOSs the $M_{\mathrm{b}}\left(\rho_{\mathrm{c}}\right)$ curve for static configurations increases monotonically up to $M_{\mathrm{b} \text {,max }}^{\text {stat }}$. The parameter $\lambda_{\max }$ gives then the maximum value of the density jump for a fixed set of other EOS parameters (adiabatic indices $\Gamma_{\mathrm{A}}$ and $\Gamma_{\mathrm{B}}$, number density threshold $n_{\mathrm{A}}$ ) for which this property of neutron stars is valid; in other words $\lambda_{\max }$ corresponds to the "marginally 
Table 2. Selected sets $\left\{K_{\mathrm{A}}, \Gamma_{\mathrm{A}}, \Gamma_{\mathrm{B}}, n_{\mathrm{A}}, \lambda\right\}$ of EOSs with a constant pressure phase transition. For all cases, $K_{\mathrm{A}}=0.025$ and $n_{\mathrm{A}}=0.25$ (see the text and Appendix A.1).

\begin{tabular}{|c|c|c|c|c|}
\hline EOS & $\lambda$ & $f_{\text {on }}[\mathrm{Hz}]$ & $M_{\mathrm{b}}^{\mathrm{on}}\left[M_{\odot}\right]$ & $M_{\mathrm{b}, \max }^{\text {stat }}\left[M_{\odot}\right]$ \\
\hline \multirow{6}{*}{$\begin{array}{l}\Gamma_{\mathrm{A}}=2, \\
\Gamma_{\mathrm{B}}=2, \\
\lambda_{\max }=1.364\end{array}$} & 1.344 & 247 & 1.033 & 1.256 \\
\hline & 1.318 & 368 & 1.071 & 1.283 \\
\hline & 1.265 & 482 & 1.131 & 1.342 \\
\hline & 1.212 & 555 & 1.193 & 1.407 \\
\hline & 1.159 & 601 & 1.248 & 1.477 \\
\hline & 1.106 & 624 & 1.286 & 1.554 \\
\hline \multirow{5}{*}{$\begin{array}{l}\Gamma_{\mathrm{A}}=2, \\
\Gamma_{\mathrm{B}}=2.25, \\
\lambda_{\max }=1.382\end{array}$} & 1.371 & 189 & 1.021 & 1.475 \\
\hline & 1.318 & 407 & 1.087 & 1.534 \\
\hline & 1.265 & 507 & 1.149 & 1.597 \\
\hline & 1.212 & 570 & 1.208 & 1.667 \\
\hline & 1.159 & 620 & 1.272 & 1.743 \\
\hline \multirow{5}{*}{$\begin{array}{l}\Gamma_{\mathrm{A}}=2, \\
\Gamma_{\mathrm{B}}=2.5, \\
\lambda_{\max }=1.393\end{array}$} & 1.371 & 257 & 1.034 & 1.712 \\
\hline & 1.318 & 453 & 1.110 & 1.776 \\
\hline & 1.265 & 522 & 1.160 & 1.844 \\
\hline & 1.218 & 576 & 1.215 & 1.918 \\
\hline & 1.159 & 608 & 1.258 & 1.999 \\
\hline \multirow{6}{*}{$\begin{array}{l}\Gamma_{\mathrm{A}}=2.25, \\
\Gamma_{\mathrm{B}}=2.25, \\
\lambda_{\max }=1.432\end{array}$} & 1.376 & 412 & 1.307 & 1.642 \\
\hline & 1.322 & 537 & 1.387 & 1.709 \\
\hline & 1.268 & 611 & 1.463 & 1.782 \\
\hline & 1.215 & 662 & 1.536 & 1.861 \\
\hline & 1.161 & 694 & 1.597 & 1.947 \\
\hline & 1.107 & 709 & 1.636 & 2.041 \\
\hline \multirow{6}{*}{$\begin{array}{l}\Gamma_{\mathrm{A}}=2.25, \\
\Gamma_{\mathrm{B}}=2.5, \\
\lambda_{\max }=1.444\end{array}$} & 1.430 & 222 & 1.242 & 1.803 \\
\hline & 1.376 & 449 & 1.325 & 1.859 \\
\hline & 1.322 & 556 & 1.403 & 1.940 \\
\hline & 1.268 & 623 & 1.477 & 2.016 \\
\hline & 1.215 & 668 & 1.545 & 2.099 \\
\hline & 1.161 & 696 & 1.602 & 2.190 \\
\hline \multirow{7}{*}{$\begin{array}{l}\Gamma_{\mathrm{A}}=2.5, \\
\Gamma_{\mathrm{B}}=2.5, \\
\lambda_{\max }=1.472\end{array}$} & 1.437 & 361 & 1.559 & 1.967 \\
\hline & 1.382 & 532 & 1.661 & 2.040 \\
\hline & 1.329 & 624 & 1.754 & 2.119 \\
\hline & 1.273 & 686 & 1.844 & 2.205 \\
\hline & 1.219 & 731 & 1.933 & 2.297 \\
\hline & 1.164 & 757 & 2.001 & 2.398 \\
\hline & 1.109 & 780 & 2.076 & 2.509 \\
\hline \multirow{6}{*}{$\begin{array}{l}\Gamma_{\mathrm{A}}=2.5, \\
\Gamma_{\mathrm{B}}=3, \\
\lambda_{\max }=1.487\end{array}$} & 1.437 & 428 & 1.590 & 2.348 \\
\hline & 1.382 & 566 & 1.689 & 2.427 \\
\hline & 1.329 & 646 & 1.871 & 2.512 \\
\hline & 1.273 & 701 & 1.870 & 2.603 \\
\hline & 1.219 & 738 & 1.945 & 2.703 \\
\hline & 1.164 & 761 & 2.011 & 2.811 \\
\hline
\end{tabular}

stable" case. Increasing $\lambda$ implies increasing softening of the EOS by the phase transition. If $\lambda>\lambda_{\max }$, the phase transition leads to the existence of an unstable branch of the nonrotating stellar configurations. This unstable branch separates stable family of neutron stars with A-phase cores from a second family of superdense neutron stars with B-phase cores: these are two distinct neutron-star families. It should be mentioned that this feature (existence of the unstable region) does not depend on rotation - the unstable branches exist also for rotating configurations (strictly speaking for any value of a total angular momentum of the star $J$ there exist a region with $\left.\left(\partial M_{\mathrm{b}} / \partial \rho_{\mathrm{c}}\right)_{J}<0\right)$. We have tested this feature (existence of or the lack of unstable regions) for very small departures from

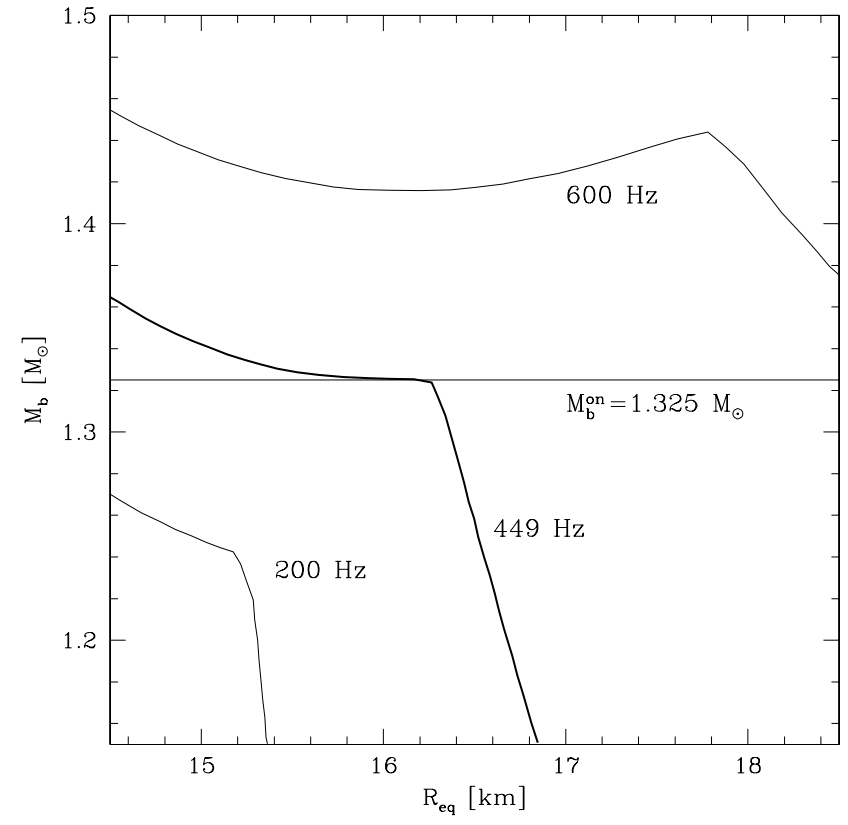

Fig. 7. The definition of the frequency of the onset of back-bending phenomenon, $f_{\mathrm{on}}$, and corresponding mass, $M_{\mathrm{b}}^{\text {on }}$. In the presented example $f_{\text {on }}=449 \mathrm{~Hz}$ and $M_{\mathrm{b}}^{\text {on }}=1.325 M_{\odot}$.

marginally stable case $\left(\left|\lambda_{\max }-\lambda\right|<0.005\right)$. From numerical results it follows that if $\lambda<\lambda_{\max }$ all rotating configurations are stable (before loosing stability at maximum mass point) and if $\lambda>\lambda_{\max }$ we have two branches of stable configuration for rotating stars (for any $J$ ).

Picking up the onset parameters is visualized in Fig. 7 where we display $M_{\mathrm{b}}\left(R_{\mathrm{eq}}\right)$ for one of the EOSs from Table 2. The curves are plotted for three frequencies, with middle one corresponding to the back bending onset, $f=f_{\text {on }}$. Last column of Table 2 gives the maximum allowable baryon mass for static configurations, $M_{\mathrm{b}, \max }^{\text {stat }}$. We restrict ourselves to back bending for the normal (non supra-massive) stars, which appears during the spin-down evolution which terminates eventually by a non-rotating stable configuration. The dependence between the back-bending onset parameters $-f=f_{\text {on }}$, corresponding baryon mass, and the intrinsic parameters of the EOS - the density jump $\lambda$, as well as the "departure" from the critical configuration $\left(\Delta \lambda=\lambda_{\max }-\lambda\right)$ is presented in Fig. 8. The three families of curves visualize the data from Table 2 (solid lines for $\Gamma_{\mathrm{A}}=2$, dotted for $\Gamma_{\mathrm{A}}=2.25$ and dashed for $\Gamma_{\mathrm{A}}=2.5$ ). The value $\lambda_{\max }$ defines the onset of back-bending at the limit $f=0$; in this case the back-bending phenomenon is present for any rotational frequency. As it can be seen on the left panel, the onset frequency $f_{\text {on }}$ depends very weakly on the EOS in the dense core - the main parameter describing the reaction of the star to the appearance of this phase transition is the density jump. The right panel presents the same data not normalized with respect to the maximum density jump $\lambda_{\max }$ - the results can be very well approximated by the dependence $f_{\text {on }}^{2}=a \Delta \lambda+b(\Delta \lambda)^{3 / 2}$. These two plots can be treated as a slice through the parameter space to search for regions of the back-bending appearance in the right panel, the back-bending is present, for a particular model, above its curve. 

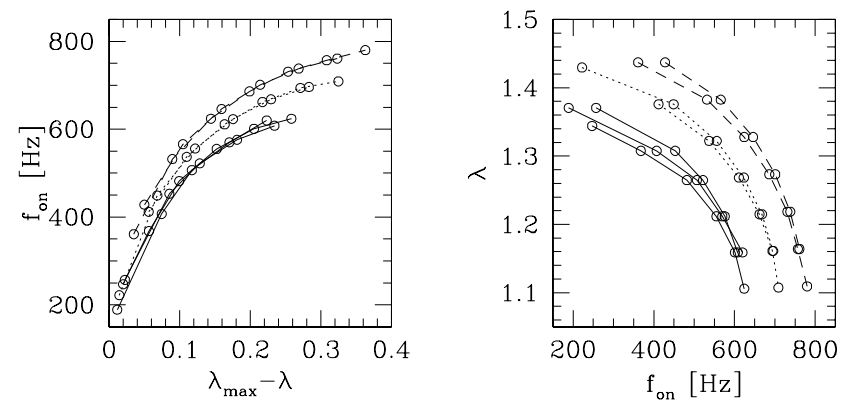

Fig. 8. The onset frequency of the back-bending phenomenon as a function of the departure of $\lambda$ from the maximum density jump $\lambda_{\max }$ $\left(\lambda_{\max }-\lambda\right.$, left panel), and density jump $\lambda$ as a function of $f_{\mathrm{on}}$ (right panel) for models presented in Table 2 (solid lines for $\Gamma_{\mathrm{A}}=2$, dotted for $\Gamma_{\mathrm{A}}=2.25$ and dashed for $\Gamma_{\mathrm{A}}=2.5$ ).

\section{Rotation and stability/instability of normal configurations}

Rotation can influence stability of a star of a given baryon mass. In particular, stars with $M_{\mathrm{b}}>M_{\mathrm{b}, \max }^{\text {stat }}$ cannot exist without rotation and collapse into black hole as soon as their rotation frequency falls below a certain minimum value. Here, however, we will restrict to a different problem of stability, which will concern the normal configurations only.

In what follows we will use the term "stability" in a restricted sense. Namely, by stability (instability) of an equilibrium configuration we will mean stability (instability) with respect to radial perturbations in the non-rotating case, and with respect to axi-symmetric perturbations for rotating configurations.

We studied a very large set of EOSs with phase transitions at constant pressure, as well as those with transition through a mixed phase state. We then produced static sequences and normal rotating sequences for these EOSs. Our calculations were very precise, because we used analytic forms of the EOSs. The results for both constant pressure phase transitions, and those proceeding through mixed phase, turned out to be qualitatively the same. In all cases, if non-rotating configuration were stable (monotonically increasing $M_{\mathrm{b}}\left(\rho_{\mathrm{c}}\right)$ and $M\left(\rho_{\mathrm{c}}\right)$ ), then for any value of the total angular momentum $J$ the functions $M_{\mathrm{b}}\left(\rho_{\mathrm{c}}\right)_{J}$ and $M\left(\rho_{\mathrm{c}}\right)_{J}$ were monotonically increasing, too. Thus, when all non-rotating configuration with $M_{\mathrm{b}}<M_{\mathrm{b} \text {,max }}^{\text {stat }}$ were stable (with respect to radial perturbations), all normal rotating configurations were stable too (with respect to axi-symmetric perturbations). On the other hand, if for non-rotating stars there existed a region with decreasing $M_{\mathrm{b}}\left(\rho_{\mathrm{c}}\right)$ and $M\left(\rho_{\mathrm{c}}\right)$, even extremely small one with a very shallow minimum, then an unstable region persisted within the rotating configurations, at each value of $J$. These two cases are illustrated in the Figs. 5 and 6.

We studied also the case of marginally stable EOS. An inflection point, witnessing marginal stability, present in the $M_{\mathrm{b}}(R)$ or $M\left(\rho_{\mathrm{c}}\right)$ curves for non-rotating stars continued to exist in the $M_{\mathrm{b}}\left(R_{\mathrm{eq}}\right)$ or $M\left(\rho_{\mathrm{c}}\right)_{J}$ curves for normal rotating stars (Figs. 4, 9).

The analysis of numerical results leads us to an interesting conclusion. Namely, for an EOS with a phase transition (constant pressure one or through mixed-phase state), rotation

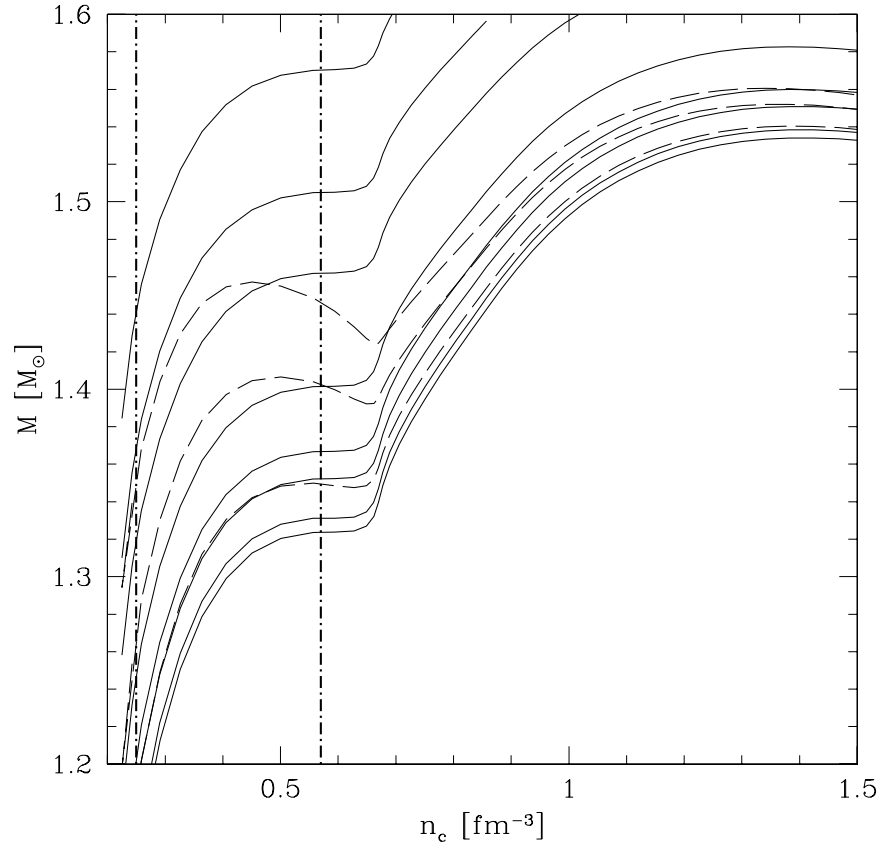

Fig. 9. The mass $M$ of the star as a function of central baryon number density $n_{\mathrm{c}}$ for the MM model. Dashed lines - fixed rotational frequency. Solid lines - fixed total angular momentum $J$. The vertical lines correspond to the densities of transitions from the nuclear to mixed phase and from the mixed phase to the pure denser phase (here - quark matter) - see Table 1.

neither stabilizes nor destabilizes normal sequences of stationary configurations based on this EOS. We define a family of configurations as a compact set (in mathematical sense) of configurations. Similarly, an EOS leading to a marginally stable point for non-rotating stars, produces also spin-down evolution tracks with a marginally stable point. Our result can be formulated as three conjectures:

I) If an EOS with a phase transition gives a single family of static stable neutron stars then it produces also a single family of stable rigidly rotating normal stars.

II) If an EOS with a phase transition gives two disjoint families of stable static stars then it gives also two disjoint families of stable rigidly rotating normal stars.

III) If an EOS with a phase transition gives two families of stable static stars separated by a marginally stable configuration, then it gives also two families of stable rigidly rotating normal stars separated by a line consisting of marginally stable configurations.

\section{Corequake resulting from instability}

As was shown on many occasions in the preceding sections, an isolated neutron star, loosing its angular momentum, moves down along the line of fixed $M_{\mathrm{b}}$ in the $J(f)$ plane, and can at some moment reach the instability point (i) (the minimum of $J(f)$ at fixed $M_{\mathrm{b}}$ in Fig. 10). The subsequent behavior of the star cannot be described by our stationary rigidly rotating model. In real world, the star has to collapse, rearranging the angular momentum distribution in its interior. What we can do, 


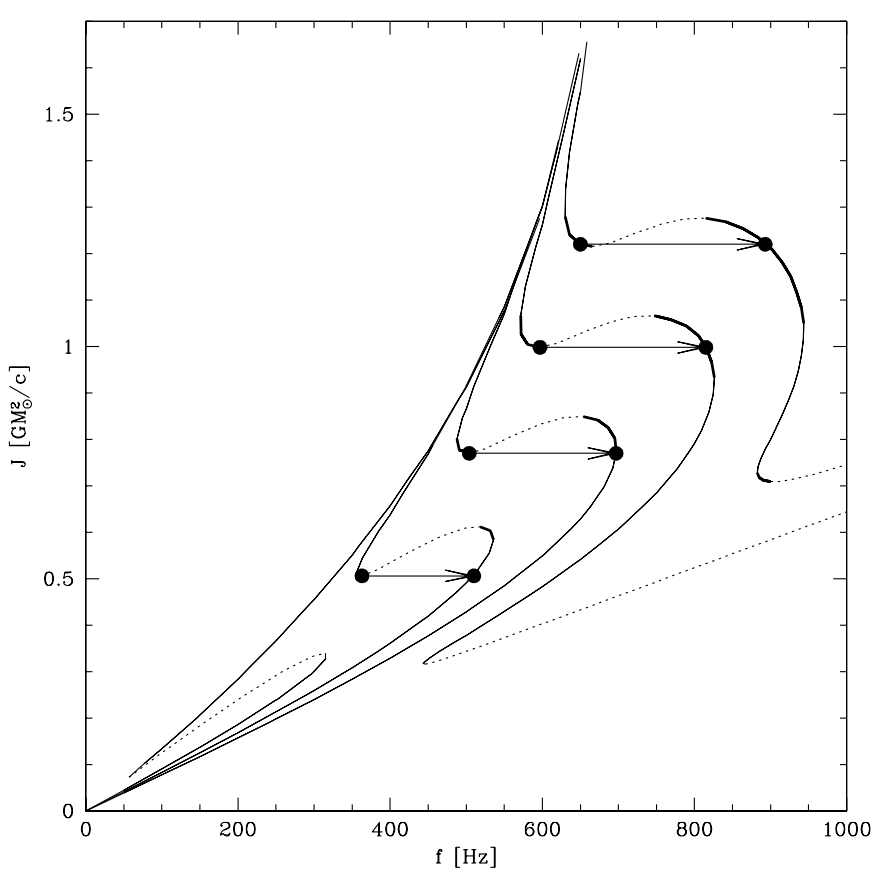

Fig. 10. Evolution of an isolated pulsar loosing angular momentum, after it reaches the instability region in $J-f$ plane and then collapses. Arrows lead from unstable configuration to a collapsed stable one, with the same baryon mass and angular momentum. Dotted lines unstable configurations.

is determining the final stationary state (f), which by construction will be a stable rigidly rotating neutron star. We assume, that the transition conserves the baryon number of the star and is sufficiently rapid so that the angular momentum loss can be neglected. Therefore, the final stable configuration will have the same $M_{\mathrm{b}}$ and $J$ as the unstable initial one, $M_{\mathrm{b}, \mathrm{f}}=M_{\mathrm{b}, \mathrm{i}}$, $J_{\mathrm{f}}=J_{\mathrm{i}}$. The difference between the parameters of these configurations (mass, equatorial radius, moment of inertia) gives us the energy release, and changes in equatorial radius and rotation frequency, due to the collapse implied by the instability.

Examples are shown in Figs. 10-12 for our EOS model MUn. As the star becomes more compact, collapse is accompanied by the decrease of the equatorial radius (by a few kilometers) and by a significant spin up. For a given EOS, the changes in radius, energy release, and spin-up are function of the angular momentum at the instability point: $\Delta R_{\mathrm{eq}}\left(J_{\mathrm{i}}\right), \Delta E\left(J_{\mathrm{i}}\right), \Delta f\left(J_{\mathrm{i}}\right)$. As we see in Fig. 11, the energy release depends rather weakly on the rotation of the unstable configuration (i.e., on the value of $J_{\mathrm{i}}$ ). It should be mentioned that our MUn model is only an example of the EOS resulting in the instability region within the hydrostatic equilibria. For EOSs with a weaker phase transition this instability region would be narrower and the changes of stellar parameters in the collapse would be smaller. However an approximate constancy of the energy release (i.e., its very weak dependence on $J=J_{\mathrm{i}}=J_{\mathrm{f}}$ ) seems to be a generic property of rotating neutron stars undergoing a collapse due to a first order phase transition.

In order to discuss in more detail the energy release during collapses $\mathrm{i} \longrightarrow \mathrm{f}$, we plotted in Fig. 12 the gravitational mass of the star, $M$, as a function of angular momentum, $J$, at

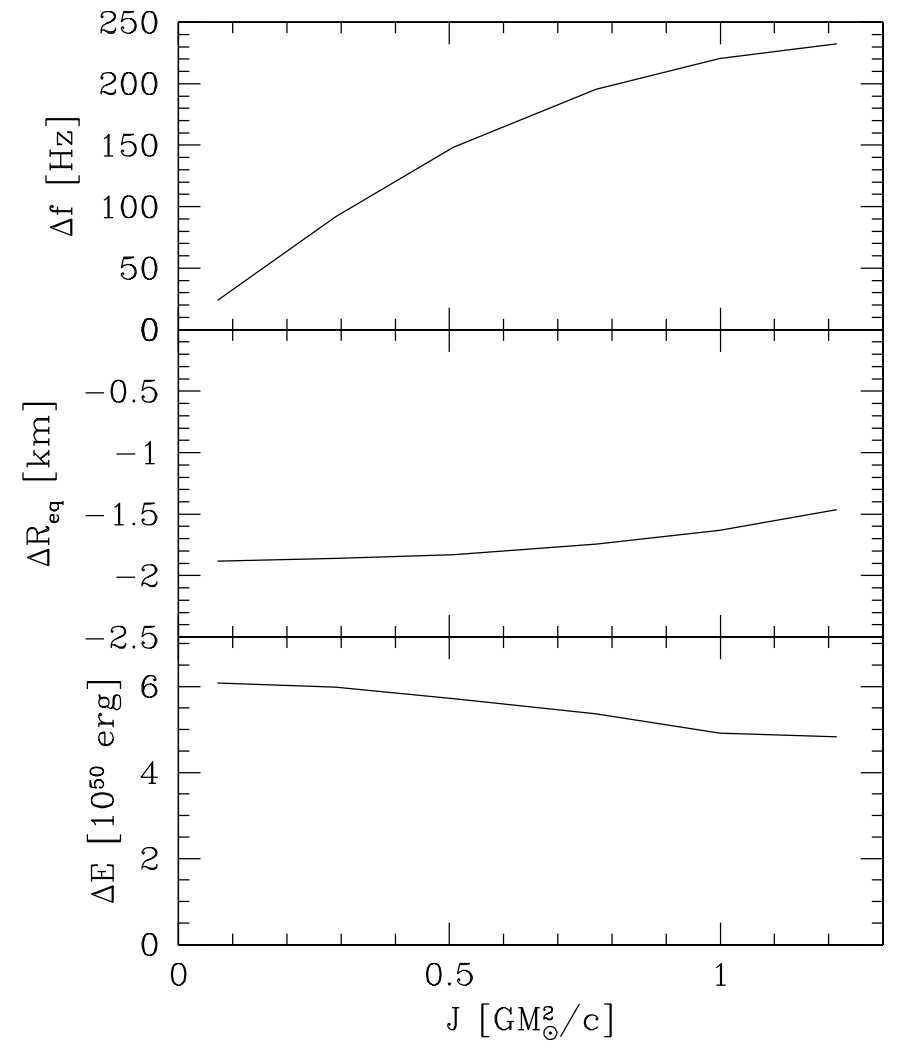

Fig. 11. Changes of stellar parameters of a rotating solitary neutron star, due to a collapse which occurs after a pulsar loosing angular momentum reaches an unstable configuration.

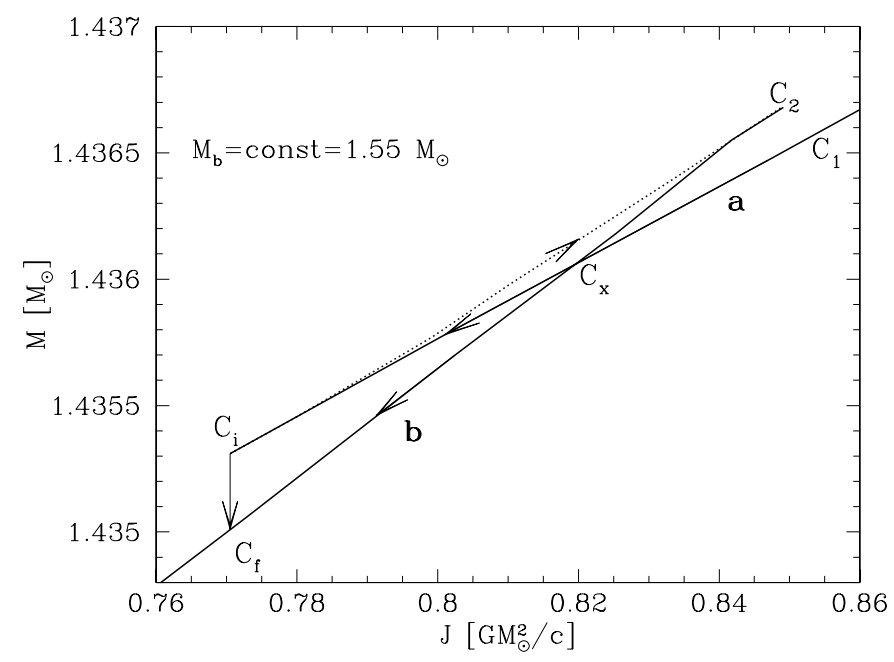

Fig. 12. Total gravitational mass of the star as a function of its angular momentum, for fixed baryon number of the star for our EOS model MUn. The central density is increasing along this curve as marked by the arrows. The upper segment (dotted) corresponds to the unstable configurations. Two cusps reflect strict property that the mass and angular momentum have simultaneous extrema along the path with fixed baryon number.

fixed baryon mass: $M=M(J)_{M_{\mathrm{b}}}$. Consider an initial configuration $C_{1}$. As the star looses angular momentum, it moves down along line $a$, and reaches eventually the cusp $C_{\mathrm{i}}$ (corresponding to the value of $J=J_{\mathrm{i}}$ ). To continue moving on the dotted 
segment $C_{\mathrm{i}} C_{2}$, the star would have to gain angular momentum and energy!

As we already mentioned, the evolution of the star beyond the instability point cannot be described by our hydrostationary model. The star can only collapse to the final configuration $C_{\mathrm{f}}$, with the same values of $M_{\mathrm{b}}$ and $J$, i.e. along vertical arrow in Fig. 12. Then, it evolves down the line $b$.

We notice a very special role played by the point $C_{\mathrm{x}}$ at which line $a$ and $b$ cross. This is a degeneracy point, which corresponds to two very different configurations of the same $M_{\mathrm{b}}$, $M$, and $J$. However, transitions between these two configuration are prevented by the huge energy barrier.

The existence of the sharp cusps at $C_{\mathrm{i}}$ and $C_{2}$ on the $M(J)_{M_{\mathrm{b}}}$ track is a very stringent test of the precision of the numerical code: it means that mass and angular momentum extrema (for fixed baryon mass) are reached exactly at the same point. This property follows from the general relativistic relation $\mathrm{d} M=$ $\Omega \mathrm{d} J+\gamma \mathrm{d} M_{\mathrm{b}}$ (Bardeen 1972). Here, $\Omega \equiv 2 \pi f$, and $\gamma$ is the "injection energy per unit mass". This relation has to be strictly fulfilled by the stationary configurations. A graph, analogous to Fig. 12, can be plotted in the $M-M_{\mathrm{b}}$ plane for configurations with fixed angular momentum $J$. Also in this case, the existence of sharp cusps proves the correctness of the numerical code.

\section{Phase transition and pulsar timing and age}

It has been already pointed out by Spyrou \& Stergioulas (2002), that the back bending phenomenon, resulting from the growth of a dense-phase core, can lead to significant difference between the actual pulsar age, $\tau_{1}$, and that inferred from the measurements of the period $P$ and period derivative, $\dot{P}$, and denoted $\tau_{2}$. The calculation of $\tau_{2}$ is based on quite strong assumptions. Firstly, pulsar kinetic energy loss due to radiation is given by the magnetic dipole formula. Secondly, non-relativistic approximation is used, with pulsar kinetic energy given by $\frac{1}{2} I \Omega^{2}$, where $\Omega \equiv 2 \pi / P$ and pulsar moment of inertia is constant, independent of $\Omega$.

Following Spyrou \& Stergioulas (2002), we will use general relativistic notion of total pulsar energy, $M c^{2}$. Then the pulsar energy balance is

$\frac{\mathrm{d} M}{\mathrm{~d} t}=-\frac{\kappa}{c^{2}} \Omega^{\alpha}$

where the right-hand-side is the pulsar energy loss rate via radiation of electromagnetic waves and particles.

Let us consider the increase of stellar energy due to a spin up to frequency $f$ at constant $M_{\mathrm{b}}$. In general relativity, the increase is given by $\Delta M(f)=[M(f)-M(0)]_{M_{\mathrm{b}}}$. In the standard model, we neglect the effect of $f$ on stellar structure, so that $\Delta M=\frac{1}{2} I(2 \pi f)^{2}$. This is a good approximation when rotation is slow and EOS is smooth (no phase transition). Equation (2) can be then rewritten as

$\dot{\Omega}=-\frac{\kappa}{I_{0}} \Omega^{\alpha-1}$,

where $I_{0} \equiv I(0)=$ const.

In the case when angular momentum loss leads to a phase transition at the stellar center, the situation is much more complicated, because of the strong $f$-dependence of the pulsar

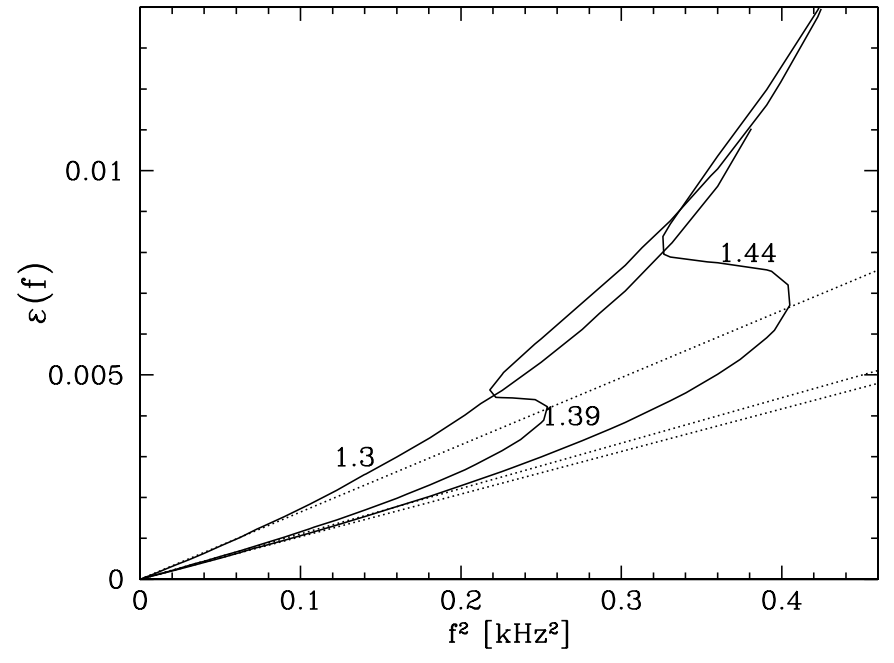

Fig. 13. The relative mass-energy increase due to rotation of the star at fixed baryon mass, $\varepsilon(f) \equiv[(M(f)-M(0)) / M(0)]_{M_{\mathrm{b}}}$, for the EOS with a phase transition (MM model), is shown using solid line. Three solid lines are labeled by the gravitational mass of the non-rotating configuration (in solar masses). Dotted lines correspond to the $\varepsilon(f)$ curves calculated for the standard model, Eq. (3), with $\alpha=4$.

structure in the vicinity of the phase transition. This difference is illustrated in Fig. 13, where we plotted the quantity $\varepsilon(f) \equiv \Delta M(f) / M(0)$ resulting from our calculations, and compared it with results given by standard non-relativistic model with constant $I$. As it can be seen in Fig. 13, the approximation given by Eq. (3) significantly overestimates the change of frequency associated with a given energy loss. This in turn can lead to an underestimation of the age of the pulsar - an example is presented in Fig. 14. There, we plot the pulsar period, $P$, as a function of time, obtained by the integration of Eq. (2) for magnetic dipole braking $(\alpha=4)$. We also show results inferred from the observation of a $10 \mathrm{~ms}$ pulsar, assuming four selected values of the pulsar baryonic mass. Taking $P=10 \mathrm{~ms}$ and corresponding $\dot{P}$ the extrapolation backward in time using standard model, Eq. (3), diverges from exact results as soon as $P<5 \mathrm{~ms}$. If the pulsar is born with period of $2 \mathrm{~ms}$, then its real age is significantly longer than $P / 2 \dot{P}$.

\section{Discussion and conclusions}

The effect of phase transitions in dense neutron-star cores on the spin-down evolution of isolated stars was studied using analytical representations of EOSs. This guaranteed a very high precision of our 2-D calculations and allowed us to study a very large parameter space as far as the phase transitions themselves are concerned. In this way, we studied a very broad class of constant-pressure phase transitions associated with a density jump in the EOS. We also studied the case of transitions through a mixed-phase state.

We limited ourselves to the case of normal rotating configurations, which are connected with the non-rotating ones by the angular momentum loss. We considered two types of instabilities which bound the sets of stationary configurations: mass shedding and instability with respect to the axisymmetric perturbations. The EOSs split into two sets: those producing a 


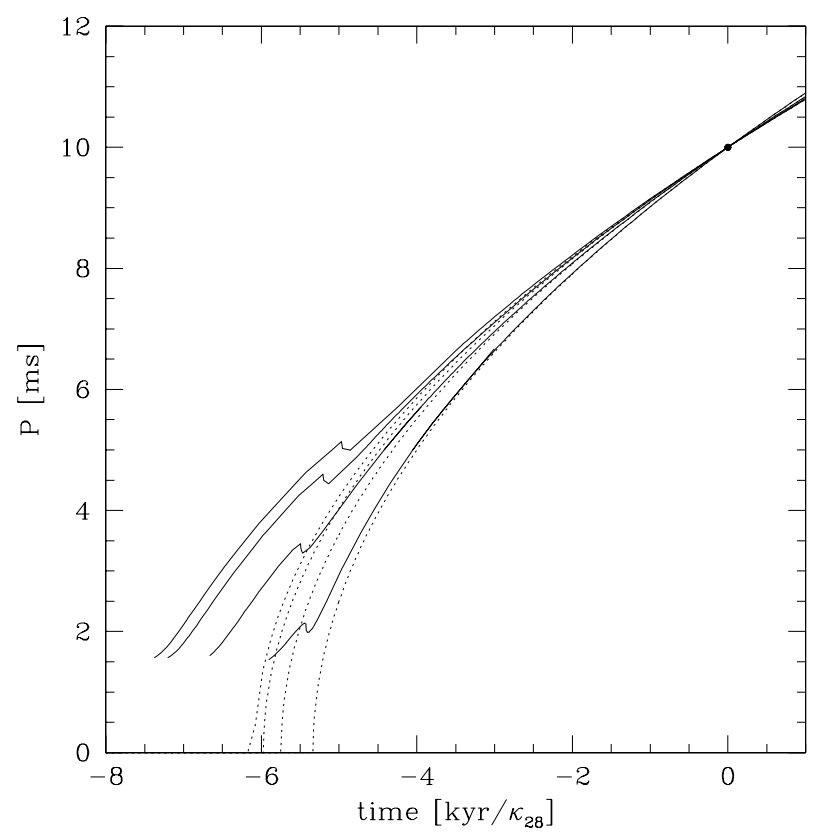

Fig. 14. The evolution of the pulsar period when the energy loss is described by the magnetic dipole braking with $\alpha=4$. Solid curves - results for our model MM with different values of baryon mass. Dotted lines correspond to the standard model, Eq. (3). The unit of time (horizontal axis) is $1000 \mathrm{yr} / \kappa_{28}$ where $\kappa_{28}=\kappa / 10^{28}$ [cgs] is parameter entering Eq. (2).

single family of stable stationary configurations (static and rotating) of neutron stars and those producing two disjoint families of stable stationary configurations. Conjectures concerning normal configurations based on EOSs with a phase transition has been formulated. If an EOS yields two disjoint families of static configurations containing "twin neutron stars" of the same baryon mass but different radius, then also stable normal rotating configurations form two disjoint regions in the mass equatorial radius plane, which contain "twin neutron stars" of the same baryon mass but of different compactness.

Very often, neutron stars are called a second family of compact stars, the first family being composed of white dwarfs. Therefore, our conjecture means that an EOS which produces a third (disjoint) family of static compact stars, produces also a third (disjoint) family of rotating normal stars.

We have also shown the existence of a very special class of "fine tuned EOSs" with phase transitions which produce marginally stable stationary configurations of normal neutron stars, which form a boundary separating stable stationary configurations (a line in the mass-equatorial radius plane).

Conditions on the density jump in constant-pressure phase transitions were derived, under which their presence in the EOS produces the back bending phenomenon in the spin down evolution.

The case when a spinning down normal neutron star reaches an unstable configuration was studied in detail. The instability leads to neutron star collapse, associated with an energy release in a "corequake", decrease of radius, increase of central density, and spin up of the star. We have shown that the energy release associated with such a "corequake" depends rather weakly on the initial rotation frequency at the instability point. In our examples, energy release was of the order of a few times $10^{50} \mathrm{erg}$.

In the present paper we put accent on the numerical precision and mathematical strictness. We hope that in this way we prepared ground for further studies of the impact of the phase transitions in dense matter on the structure, evolution, and dynamics of rotating neutron stars. These further studies will be performed using realistic EOSs available in the literature and taking into account important microscopic aspects of the phase transitions. The kinetics of the phase transition coupled with stellar spin-down, and the ensuing neutron star corequake are now being studied. These topics will be the subject of our subsequent papers.

Acknowledgements. This work was partially supported by the Polish MNiI grant no. 1P03D-008-27 and by the PAN/CNRS LEA Astro-PF.

\section{Appendix A: Analytical equations of state}

The relativistic polytrope, following Tooper (1965), relates pressure $p$ to baryon number density $n_{\mathrm{b}}$ by

$p\left(n_{\mathrm{b}}\right)=K n_{\mathrm{b}}{ }^{\Gamma}$

where $\Gamma$ is the adiabatic index. The second coefficient, $K$, is often called the pressure coefficient ${ }^{1}$.

Dense matter is strongly degenerate, so that the $T=0$ approximation is valid. First Law of Thermodynamics implies then expression for energy per baryon

$\mathcal{E} / n_{\mathrm{b}}=\frac{K n_{\mathrm{b}}^{\Gamma-1}}{\gamma-1}+C$

where the constant $C$ is set, for $n_{\mathrm{b}}=0$, to be the unit baryon rest energy ${ }^{2} C=m_{0} c^{2}$.

The energy density $\mathcal{E}$ is thus given by

$\mathcal{E}\left(n_{\mathrm{b}}\right)=\frac{K}{\Gamma-1} n_{\mathrm{b}}^{\gamma}+m_{0} c^{2} n_{\mathrm{b}}$

The baryon chemical potential $\mu$ is therefore

$\mu\left(n_{\mathrm{b}}\right)=\frac{p+\mathcal{E}}{n_{\mathrm{b}}}=\frac{\Gamma K}{\Gamma-1} n_{\mathrm{b}}^{\Gamma-1}+m_{0} c^{2}$

\section{A.1. Constant pressure phase transition}

We assume that the phase transition takes place in thermodynamic equilibrium. In a simplest case the transition from less dense pure phase A to high-density pure phase B occurs at constant pressure. Example of such transition is shown in Fig. A.1. Pure phases, $\mathrm{A}$ and $\mathrm{B}$, will be approximated by polytropes, with adiabatic indices $\Gamma_{A}$ and $\Gamma_{B}$, respectively. Let us also fix the mean baryon mass $m_{0 \mathrm{~A}}$ and coefficient $K_{\mathrm{A}}$, and demand that the

\footnotetext{
1 Unless otherwise mentioned, the coefficient $K$ will be measured in $\hat{\rho} c^{2} / \hat{n}^{\Gamma}$ units, where $\hat{\rho}:=1.66 \times 10^{14} \mathrm{~g} / \mathrm{cm}^{3}$, and $\hat{n}:=0.1 \mathrm{fm}^{-3}$.

2 Following many authors, we put unit baryon mass equal to mass per nucleon in the ground state of atomic matter at zero pressure, which is ${ }^{56} \mathrm{Fe}$ crystal, $m_{0}=1.66 \times 10^{-24} \mathrm{~g}$.
} 

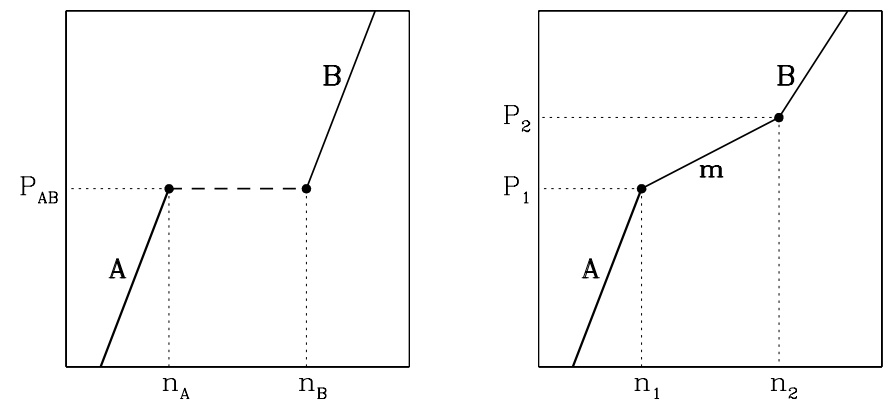

Fig. A.1. Examples of phase transitions considered in the text; constant pressure phase transition (left), and the phase transition through the mixed-phase state (right).

phase transition occurs between the baryon densities $n_{\mathrm{A}}$ and $n_{\mathrm{B}}$, at constant pressure $\mathrm{P}_{\mathrm{AB}}$ and chemical potential $\mu$. From constancy of pressure and chemical potential we get

$$
\begin{aligned}
& K_{\mathrm{B}}=K_{\mathrm{A}} \cdot n_{\mathrm{A}} \Gamma_{\mathrm{A}} / n_{\mathrm{B}} \Gamma_{\mathrm{B}}, \\
& m_{0 \mathrm{~B}}=m_{0 \mathrm{~A}}+\mathrm{P}_{\mathrm{AB}}\left(\frac{\Gamma_{\mathrm{A}}}{n_{\mathrm{A}}\left(\Gamma_{\mathrm{A}}-1\right)}-\frac{\Gamma_{\mathrm{B}}}{n_{\mathrm{B}}\left(\Gamma_{\mathrm{B}}-1\right)}\right) .
\end{aligned}
$$

\section{A.2. Transition through the mixed-phase state}

Lower-density phase $\mathrm{A}$ extends up to pressure $\mathrm{P}_{1}$ and density $n_{\mathrm{b}}=n_{1}$, then follows mixed m-phase with volume fraction of dense phase $\chi_{\mathrm{B}}$ increasing monotonically from 0 at $n_{1}$ to 1 at $n_{\mathrm{b}}=n_{2}$ and $p=\mathrm{P}_{2}$. Again, for simplicity, it will be assumed that the phase A and mixed-phase $m$ can be approximated by polytropes (not a bad approximation, see Bejger et al. 2005). The parameters $K_{\mathrm{m}}, \Gamma_{\mathrm{m}}$, and $m_{0 \mathrm{~m}}$ (the mean particle mass in the mixed-phase) must be related to those of the A-phase in such a way that pressure and baryon chemical potential stay continuous across the $\mathrm{A} \longrightarrow \mathrm{m}$ transition point at $n_{1}$,

$$
\begin{aligned}
& K_{\mathrm{m}}=K_{\mathrm{A}} \cdot n_{1} \Gamma_{\mathrm{A}}-\Gamma_{\mathrm{m}} \\
& m_{0 \mathrm{~m}}=m_{0 \mathrm{~A}}-\frac{\mathrm{P}_{1}}{n_{1} c^{2}} \cdot \frac{\Gamma_{\mathrm{A}}-\Gamma_{\mathrm{m}}}{\left(\Gamma_{\mathrm{A}}-1\right)\left(\Gamma_{\mathrm{m}}-1\right)} .
\end{aligned}
$$

We will further assume that the high-density phase $\mathrm{B}$, existing at $n_{\mathrm{b}}>n_{2}, p>\mathrm{P}_{2}$, and $\rho>\rho_{2}$, is pure quark matter, with MIT bag model EOS (Zdunik 2000),

$p(\mathcal{E})=\frac{1}{3}\left(\mathcal{E}-\mathcal{E}_{0}\right), \quad n_{\mathrm{b}}(p)=n_{0}(1+p / \mathcal{E})^{3 / 4}$,

where $\mathcal{E}_{0}=\rho_{0} c^{2}$ and $n_{0}$ are the mass-energy density and the baryon density of the quark matter at zero pressure. The baryon chemical potential of the B-phase is then equal to

$\mu(p)=\mu_{0}(1+4 p / \mathcal{E})^{1 / 4}$,

where $\mu_{0}=\mathcal{E}_{0} / n_{0}$.
From the continuity of pressure, baryon density, and energy density at the $\mathrm{m} \longrightarrow \mathrm{B}$ transition point, we get

$$
\begin{aligned}
\mathcal{E}_{0} & =\mathcal{E}_{2}-3 \mathrm{P}_{2}\left(n_{2}\right), \\
n_{0} & =n_{2} /\left(1+4 \mathrm{P}_{2} / \mathcal{E}_{2}\right)^{3 / 4} .
\end{aligned}
$$

\section{References}

Balberg, S., \& Gal, A. 1997, Nucl. Phys. A, 625, 435

Bardeen, J. M. 1972, in Black Holes, ed. C. De Witt (New York: Gordon and Breach), 260

Bejger, M., Haensel, P., \& Zdunik, J. L. 2005, MNRAS, 359, 699

Bonazzola, S., Gourgoulhon, E., Salgado, M., \& Marck, J.-A. 1993, A\&A, 278, 421

Bonazzola, S., Gourgoulhon, E., \& Marck, J.-A. 1998, Phys. Rev. D, 58,104020

Chubarian, E., Grigorian, H., Poghosyan, G., \& Blaschke, D. 2000, A\&A, 357, 968

Cheng, K. S., Yuan, Y. F., \& Zhang, J. L. 2002, ApJ, 564, 909

Friedman, J. L., Ipser, J. R., \& Sorkin, R. D. 1988, ApJ, 325, 722

Glendenning, N. K. 1992, Phys. Rev. D, 46, 1274

Glendenning, N. K. 2000, Compact Stars, Nuclear Physics, Particle Physics and General Relativity (New York: Springer-Verlag)

Glendenning, N. K., Pei, S., \& Weber, F. 1997, Phys. Rev. Lett., 79, 1603

Gourgoulhon, E., Haensel, P., Livine, R., Paluch, E., Bonazzola, S., \& Marck, J.-A. 1999, A\&A, 349, 851

Hartle, J. B. 1967, ApJ, 150, 1005

Hartle, J. B., \& Thorne, K. S. 1968, ApJ, 153, 807

Heiselberg, H., \& Hjorth-Hensen, M. 1998, Phys. Rev. Lett., 80, 5485

Komatsu, H., Eriguchi, Y., \& Hachisu, I. 1989a, MNRAS, 237, 355

Komatsu, H., Eriguchi, Y., \& Hachisu, I. 1989b, MNRAS, 239, 153

Nozawa, T., Stergioulas, N., Gourgoulhon, E., \& Eriguchi, Y. 1998, A\&AS, 132, 431

Ring, P., \& Schuck, P. 1980, The Nuclear Many Body Problem (Berlin: Springer)

Salgado, M., Bonazzola, S., Gourgoulhon, E., \& Haensel, P. 1994, A\&A, 291, 155

Spyrou, N. K., \& Stergioulas, N. 2002, A\&A, 395, 151

Stergioulas, N., \& Friedman, J. L. 1995, ApJ, 444, 306

Tooper, R. F. 1965, ApJ, 142, 1541

Weber, F., \& Glendenning, N. K. 1991, Phys. Lett. B, 265, 1

Weber, F., \& Glendenning, N. K. 1992, ApJ, 390, 541

Zdunik, J. L. 2000, A\&A, 359, 311

Zdunik, J. L., Haensel, P., Gourgoulhon, E., \& Bejger, M. 2004, A\&A, 416, 1013

Zdunik, J. L., Haensel, P., \& Schaeffer, R. 1987, A\&A, 172, 95 